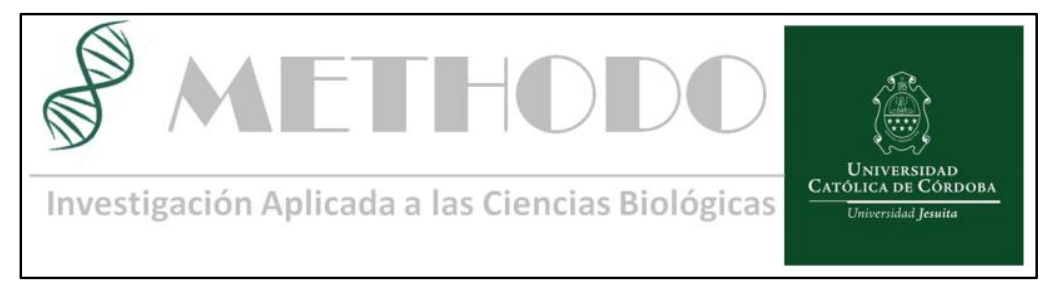

ARTICULO ORIGINAL Methodo 2018;3(4)103 - 111 DOI: $10.22529 / \mathrm{me} .2018 .3(4) 02$

\title{
Rol de variantes genéticas en el desarrollo de Enfermedad Renal Crónica en pacientes con Diabetes Mellitus Tipo 2.
}

\section{Role of genetic variants to the development of Chronic Kidney Disease in patients with Type 2 Diabetes Mellitus.}

\author{
Pablo Yang ${ }^{1}$, Verónica Ojeda, ${ }^{2}$ Adriana Ruiz-Pecchio. ${ }^{3}$, Néstor W. Soria ${ }^{1}$
}

\begin{abstract}
1 Cátedra de Biotecnología, Facultad de Ciencias Químicas, Universidad Católica de Córdoba. Unidad Asociada al CONICET: Área de Cs. Agrarias, Ingeniería, Cs. Biológicas. Obispo Trejo 323. CP: 5000IYG. Córdoba. Córdoba. Argentina. TE: + 543514938060.

2 Departamento de Diabetología, Hospital Nacional de Clínicas, Universidad Nacional de Córdoba. Santa Rosa 1564. CP: 5000ETF. Córdoba. Córdoba. Argentina. 3 Laboratorio de Nefrología, Laboratorio Central, Hospital Nacional de Clínicas, Universidad Nacional de Córdoba. Santa Rosa 1564. CP: 5000ETF. Córdoba. Córdoba. Argentina.

Correspondencia: Néstor W. Soria, Cátedra de Biotecnología, Facultad de Ciencias Químicas, Universidad Católica de Córdoba. Unidad Asociada al CONICET: Área de Cs. Agrarias, Ingeniería, Cs. Biológicas. Obispo Trejo 323. CP: X5000DHK. Córdoba. Córdoba. Argentina; e-mail: nestorwsoria@ gmail.com
\end{abstract}

\section{Resumen}

La Enfermedad Renal Crónica (ERC) es una afección que perjudica a un gran número de pacientes. Una de las causas es la Nefropatía en pacientes con Diabetes Mellitus Tipo 2 (DM2).

Objetivo: Indagar si las presencias de variantes genéticas contribuyen al desarrollo de ERC en pacientes con DM2.

Materiales y métodos: se evaluaron criterios clínicos, bioquímicos y moleculares en 25 pacientes con DM2. Los polimorfismos se analizaron mediante PCR-RFLP para ECA (rs4646994); CDKAL1 (rs7756992); e-NOS (rs1799983) y SLC12A3 (rs11643718).

Resultados: El análisis estadístico mediante modelo dominante arrojaron para: ECA (rs4646994) (OR=1,33 (IC 95\%) 0,25-7,01; $\mathrm{p}=0,73)$; CDKAL1 (rs7756992) $(\mathrm{OR}=1(\mathrm{IC} 95 \%)$ 0,14-7,39; p=NA); e-NOS (rs1799983) (OR=0,29 (IC 95\%) $0,05-1,57 ; \quad \mathrm{p}=0,14)$ y SLC12A3 $(\mathrm{rs} 11643718(\mathrm{OR}=1,62(\mathrm{IC} 95 \%)$ 0,19-13,93; $\mathrm{p}=0,66)$.

Conclusiones: ninguna de las variantes evaluadas en los genes ECA, CDKAL1, e-NOS y SLC12A3 mostraron asociaciones positivas o negativas con el riesgo a desarrollar ERC en pacientes con DM2.

\begin{abstract}
Chronic Kidney Disease (CKD) is a condition that affects a large number of patients. One cause is the Nephropathy in Type 2 Diabetes Mellitus (DM2).patients.

Objective: Evaluate if some polymorphisms contribute to CKD risk in DM2 patients.

Materials and methods: clinical, biochemical and molecular parameters from 25 patients with DM2 were evaluated. The polymorphisms were analyzed by PCR-RFLP for the ECA (rs4646994); CDKAL1 (rs7756992); e-NOS (rs1799983) and SLC12A3 (rs11643718) genes.

Results: Statistical analysis using a dominant model showed: ACE (rs4646994) $(\mathrm{OR}=1.33$ $(95 \%$ CI $) \quad 0.25-7.01, \quad \mathrm{p}=0.73)$; CDKAL1 $(\mathrm{rs} 7756992)(\mathrm{OR}=1(95 \% \mathrm{CI}) 0.14-7.39 ; \mathrm{p}=\mathrm{NA})$; e-NOS (rs1799983) $(\mathrm{OR}=0.29(95 \% \mathrm{CI}) 0.05-$ 1.57; $\mathrm{p}=0.14$ ) and SLC12A3 (rs11643718 (OR = $1.62(95 \%$ CI) 0.19-) 13.93, p = 0.66). Conclusions: none of the evaluated variants in ACE, CDKAL1, e-NOS and SLC12A3 genes showed positive or negative associations with CKD risk in DM2 patients.
\end{abstract}




\section{Introducción}

La Enfermedad Renal Crónica (ERC) se define como alteraciones de la estructura o de la función del riñón que duran al menos 3 meses y que tienen implicancias para la salud, traduciéndose en una Tasa de Filtrado Glomerular estimada (TFGe) inferior a $60 \mathrm{ml} / \mathrm{min} / 1,73 \mathrm{~m} 2 .{ }^{1}$ La Diabetes Mellitus (DM) es la enfermedad que más contribuye a la ERC en todo el mundo junto con la hipertensión arterial (HTA). ${ }^{2}$

En 2017, la DM afectaba a 451 millones de pacientes en todo el mundo y se espera que incremente a 693 millones para $2045 .{ }^{3}$ Una de sus complicaciones es la Nefropatía Diabética (ND), la cual contribuye significativamente con la ERC. ${ }^{4}$

El riesgo a desarrollar ND ha sido ampliamente estudiado, existiendo algunas variantes genéticas que pueden contribuir al desarrollo de esta complicación .5,6

A partir de los hallazgos realizados en estudios de asociación génica a nivel mundial, muchos autores están buscando posibles relaciones entre la presencia de variantes genéticas y la manifestación de una patología o de una complicación de la misma. En nuestro país, existen escasos estudios genéticos, particularmente en el área de la ERC.

En parte como consecuencia de lo mencionado anteriormente, el motivo de nuestro estudio fue realizar un trabajo piloto en un grupo de pacientes con DM Tipo 2 (DM2) de la ciudad de Córdoba, Argentina, a los fines de evaluar el riesgo a desarrollar ERC según la presencia de los SNPs: rs4646994 $7, \quad r s 7756992{ }^{8}, \quad r s 17999833^{9} \mathrm{y}$ rs11643718 ${ }^{10}$ en cuatro genes diferentes (ECA; CDKAL1; e-NOS y SLC12A3).

\section{Materiales y métodos}

\section{Pacientes y evaluación clínica}

Se reclutaron un total de 25 pacientes con DM2 de entre 53 y 86 años de edad (11 hombres y 14 mujeres) que asistieron al Hospital Nacional de Clínicas de Córdoba Capital, Argentina, entre los meses de marzo y septiembre del año 2017. El estudio fue aprobado por el Comité de Ética del Hospital Nacional de Clínicas de Córdoba y el Ministerio de Salud de Córdoba y está de acuerdo con la declaración de Helsinki. Todos los pacientes fueron informados y su consentimiento fue obtenido.

El diagnóstico de DM2 fue realizado de acuerdo a los criterios de la Asociación Americana de Diabetes de 2005.

Los pacientes fueron clasificados en dos grupos de acuerdo a su categoría ERC (con más de 60 $\mathrm{ml} / \mathrm{min} / 1,73 \mathrm{~m} 2$ de TFGe (categorías G1 y G2) y los que tenían menos de $59 \mathrm{ml} / \mathrm{min} / 1,73 \mathrm{~m} 2$ de TFGe (categorías G3a, G3b, G4 y G5)) siguiendo las guías KDIGO $2018^{11}$.

\section{Análisis de Laboratorio}

Se obtuvieron muestras de sangre venosa en ayuno de 12 horas. Las determinaciones incluyeron glucosa, hemoglobina glicosilada (HbA1c), colesterol total, triglicéridos, colesterol de baja densidad (LDL) y colesterol de alta densidad (HDL), aspartato aminotransferasa (AST), alanino aminotransferasa (ALT), gama glutamil transpeptidasa (GGT) y albuminuria $\left(1^{\circ}\right.$ orina de la mañana), todos determinados por un sistema automatizado con reactivos para biomarcadores de rutina con el equipo Roche COBAS® 6000 (Analizador Roche Diagnostics, Rotkreuz, Suiza). La Creatininemia fue cuantificada por el método cinético de Jaffé modificado y normalizado a espectrometría de masa/dilución isotópica (IDMS).

\section{Genotipado}

\section{Extracción del ADN}

El ADN de los pacientes fue obtenido a partir de sangre periférica utilizando el kit de purificación Wizard Genomic DNA (Promega, Madison, Estados Unidos) siguiendo las instrucciones del fabricante.

\section{Detección de los Polimorfismos}

Se estudiaron los polimorfismos en los genes: ECA (rs4646994); CDKAL1 (rs7756992); e-NOS (rs1799983) y SLC12A3 (rs11643718), mediante PCR-RFLP (Reacción en Cadena de la Polimerasa - Longitud del Fragmento de Restricción del Polimorfismo) usando cebadores específicos en un termociclador Biometra UNO II (Göttingen, Alemania) (Tabla 1).

Los productos de PCR se digirieron con endonucleasas de restricción específicas y los fragmentos de ADN fueron separados mediante electroforesis en gel de agarosa $(2,5 \% \mathrm{p} / \mathrm{v})$ preteñidos con bromuro de estudio y visualizados mediante irradiación con luz ultravioleta. En la Tabla 1 se encuentran detallados los genes y los polimorfismos estudiados, los cebadores $y$ enzimas de restricción empleadas y los fragmentos de ADN esperados post digestión enzimática. 


\section{Análisis Estadístico}

Se utilizó el software SNPStats (http://bioinfo.iconcologia.net/SNPstats) y los datos fueron analizados mediante un modelo de regresión logística múltiple (codominante, dominante, recesivo, sobredominante y logaditivo) para obtener los Odds-Ratios (OR), considerando una diferencia estadísticamente significativa la presencia de un valor $\mathrm{p}<0.05$, con un intervalo de confianza del $95 \%$.

Las frecuencias genotípicas y alélicas observadas de los diferentes polimorfismos se compararon con las esperadas de acuerdo con la Ley HardyWeinberg.

Los parámetros bioquímicos y clínicos se analizaron utilizando la prueba $t$ y $\chi^{2}$ espectivamente, utilizando el programa Infostat.

\section{Aspectos éticos}

\section{Resultados}

La población estudiada consistió en 25 pacientes con DM2, 15 pacientes con bajo y 10 pacientes con alto riesgo de ERC. Como se muestra en la Tabla 2 , los grupos comparados en nuestro estudio no mostraron diferencias significativas en términos de edad, sexo, duración de la enfermedad, tabaquismo, presencia de hipertensión arterial (diastólica), terapia con insulina, índice de masa corporal y albuminuria (excepto la creatininemia). Por el contrario, hay una diferencia significativa en la presión sistólica, donde los pacientes con riesgo más elevado de ERC presentaban valores más altos.

En la Tabla 3 se presentan las frecuencias genotípicas y alélicas de los cuatro genes analizados, cada uno con su correspondiente SNP. Las mismas fueron calculadas y comparadas para ver si estaban en equilibrio con la ley de HardyWeinberg, para determinar la aleatoriedad de la población tomada. Como se puede observar, en todos los casos el valor de $\mathrm{p}$ es superior a 0,05 , indicando que no hay diferencia significativa en los resultados, por lo tanto, la población estudiada no presenta ningún sesgo en su reclutamiento.

Los resultados del análisis de regresión logística múltiple se muestran en la Tabla 4 para los cuatro polimorfismos, ECA (rs4646994); CDKAL1 (rs7756992); e-NOS (rs1799983) y SLC12A3 (rs11643718). Los análisis incluían a los modelos codominante, dominante, recesivo, sobredominante y log-aditivo. En ninguno de los análisis se observaron valores de OR y $p$ significativos, lo que significa que ninguna de las variantes analizadas en la población estudiada parece influir con el riesgo a desarrollar ERC.

\section{Discusión}

El incremento de la prevalencia de la DM contribuirá a un incremento de la ND y esta, al desarrollo de ERC. Actualmente, la presencia de ERC varía a nivel global, desde el 7 al 8\% en Asia del Sur y África hasta el 11-12\% en Norteamérica, América del Sur y Europa ${ }^{12}$.

En América Latina, la prevalencia e incidencia de la ERC ha aumentado en todos los países, sin embargo, existen amplias variaciones entre las tasas de los diferentes países. En toda la región, la ERC aumentó de 119 pacientes por millón de habitantes (ppm) en 1991 a 568 ppm en 2008. Las tasas de prevalencia más altas se registraron en Puerto Rico (1170 ppm), Uruguay (1079 ppm) y Chile (1036 ppm). Las tasas de prevalencia más bajas se informaron en Nicaragua (35 ppm) y Paraguay $(92 \mathrm{ppm})^{13}$. Escasos son los datos oficiales que se tienen respecto al número de afectados en la población Argentina.

Por otra parte, como se mencionó anteriormente, la DM sigue siendo la causa principal de ERC en América Latina, con las tasas más altas en Puerto Rico (65\%), México (51\%), Venezuela (42\%) y Colombia $(42 \%)$. Las tasas más bajas se encuentran en Brasil (25,7\%), Uruguay (22,1\%), Costa Rica $(20 \%)$ y Paraguay $(15 \%)^{13}$.

Nuestro estudio, clasificó a los pacientes en dos grupos, uno con bajo riesgo y otro con alto riesgo de ERC de acuerdo a los criterios KDIGO. Entre ambos grupos de pacientes, prácticamente no había diferencia estadística en los parámetros clínicos y bioquímicos analizados, aunque las diferencias encontradas se observaron exclusivamente en la presión arterial sistólica y en la creatininemia. Como señalábamos anteriormente, es sabido que la elevada presión sistólica contribuye a un riesgo aumentado a desarrollar ERC, por lo tanto, los valores encontrados en nuestro grupo de pacientes están perfectamente de acuerdo con lo que se encuentra en otros estudios.

El polimorfismo rs4646994 del gen ECA, fue analizado por Shanmuganathan y colaboradores ${ }^{14}$. Ellos encontraron que la variante D, estaba asociada con un aumento del riesgo a desarrollar ERC. En sentido contrario, Jayapalan y colaboradores no pudieron observar esta asociación $^{15}$.

Los estudios que muestran la asociación del polimorfismo rs7756992 del gen CDKAL1 con la ERC en pacientes con DM2 son inconsistentes, algunos muestran posibles asociaciones, otros no ${ }^{16}$. 
Cuando analizamos distintos estudios para ver la contribución al desarrollo de ERC, algunos trabajos arrojan conclusiones diferentes en relación al polimorfismo rs1799983 presente en el gen e-NOS, por ejemplo el de MrozikiewiczRakowska y colaboradores, no lograron encontrar ninguna asociación ${ }^{17}$, mientras Chand y colaboradores si mostraron la existencia de una asociación ${ }^{18}$.

Por último, cuando analizamos el polimorfismo rs11643718 en el gen SLC12A3, el mismo está asociado al riesgo de desarrollar ERC en pacientes con ND en el estudio publicado por Kim y colaboradores $^{19}$. Contrariamente a estos hallazgos, $\mathrm{Ng}$ y colaboradores no encontraron ninguna asociación $^{20}$.

A partir de nuestros resultados, no podemos afirmar que alguno de los 4 polimorfismos estudiados confiere mayor o menor riesgo a desarrollar ERC. Si bien, el número de pacientes estudiados es pequeño (siendo una de las limitaciones de nuestro estudio), es importante conocer más cuáles son los factores que pueden contribuir a la ERC en pacientes con DM2, y esto utilizarlo a la hora de proyectar el desarrollo de un estudio que incluya un mayor número de pacientes. Además, según nuestras búsquedas bibliográficas, la información disponible y confiable en relación a la prevalencia e incidencia de ERC en Argentina es escasa, y este pequeño estudio puede contribuir como punto de partida, para otros proyectos que permitan darnos una idea más acabada de lo que sucede en nuestro país.

Una segunda limitación, es que el estudio fue realizado en una población de origen caucásico, por lo tanto, diferencias se podrían encontrar si se analizaran pacientes provenientes de otras regiones de nuestro país y/o la de otros países.

\section{Bibliografía}

1. Martínez-Castelao A, Górriz JL, Bover J, Segura-de la Morena J, et al. Documento de consenso para la detección y manejo de la enfermedad renal crónica. Semer - Med Fam [Internet]. 2014 Nov;40(8):441-59. Available from:

http://linkinghub.elsevier.com/retrieve/pii/S1 575092214001715

2. Monhart V. Hypertension and chronic kidney diseases. Cor Vasa [Internet]. 2013 Aug;55(4): e397-402. Available from: http://linkinghub.elsevier.com/retrieve/pii/S0 010865013000945

3. Cho NH, Shaw JE, Karuranga S, Huang Y, et al. IDF Diabetes Atlas: Global estimates of diabetes prevalence for 2017 and projections for 2045. Diabetes Res Clin Pract [Internet]. 2018 Apr; 138:271-81. Available from: http://dx.doi.org/10.1016/j.diabres.2018.02.0 23

4. Maric C, Hall JE. Obesity, Metabolic Syndrome and Diabetic Nephropathy. In: Contrib Nephrol [Internet]. 2011. p. 28-35. Available from: https://www.karger.com/Article/FullText/324 941

5. Rizvi S. Association of genetic variants with diabetic nephropathy. World J Diabetes [Internet]. 2014 Dec 15 [cited 2015 Aug 9];5(6):809-16. Available from: http://www.pubmedcentral.nih.gov/articleren der.fcgi?artid=4265867\&tool=pmcentrez\&re ndertype $=$ abstract

6. Tamm R, Oselin K, Kallassalu K, Magi R, et al. Thiopurine S-methyltransferase (TPMT) pharmacogenetics: three new mutations and haplotype analysis in the Estonian population. Clin Chem Lab Med [Internet]. 2008 Jan 1 [cited 2014 Jun 4];46(7):974-9. Available from:

http://www.ncbi.nlm.nih.gov/pubmed/18605 963

7. Fathi M, Nikzamir AR, Esteghamati A, Nakhjavani M, et al. Combination of Angiotensin Converting Enzyme Insertion/Deletion (I/D) (rs4646994) and VEGF Polymorphism (+405G/C; rs2010963) Synergistically Associated With the Development, of Albuminuria in Iranian Patients With Type 2 Diabetes. Iran Red Crescent Med J [Internet]. 2015 Feb 21 [cited 2015 Oct 22];17(2): e19469. Available from: http://www.pubmedcentral.nih.gov/articleren der.fcgi ?artid=4376980\&tool=pmcentrez\&re ndertype $=$ abstract

8. Li Y, Wang L, Lu X, Yang Z, et al. CDKAL1 gene rs7756992 A/G polymorphism and type 2 diabetes mellitus: a meta-analysis of 62,567 subjects. Sci Rep [Internet]. 2013 Dec 4 [cited 2014 Jul 31];3(1):3131. Available from: http://www.pubmedcentral.nih.gov/articleren der.fcgi? artid=3816287\&tool=pmcentrez\&re ndertype $=$ abstract

9. Dellamea B, Leitão C, Friedman R, Canani L. Nitric oxide system and diabetic nephropathy. Diabetol Metab Syndr. 2014;6(1):17.

10. Zhang R, Zhuang L, Li M, Zhang J, et al. Arg913Gln of SLC12A3 gene promotes development and progression of end-stage renal disease in Chinese type 2 diabetes mellitus. Mol Cell Biochem. 2018;437(1- 
2):203-10. Available from: http://link.springer.com/10.1007/s11010-0173120-z

11. Ronco P, Wong G, Agarwal A, Anders H, et al. KDIGO 2018 Clinical Practice Guideline for the Prevention, Diagnosis, Evaluation, and Treatment of Hepatitis C in Chronic Kidney Disease. Kidney Int Suppl [Internet]. 2018 Oct;8(3):91-165. Available from: https://linkinghub.elsevier.com/retrieve/pii/S 2157171618300054

12. Correa-Rotter R, García-García G, Iñiguez JC, Ramirez-Sandoval JC. Ethnicity and Chronic Kidney Disease in Disadvantaged Populations - An International Perspective. In: Chronic Renal Disease. Elsevier; 2015. p. 833-42.

13. Kumaresan R, Giri P, Shanmuganathan R. Prevalence of angiotensin converting enzyme (ACE) gene insertion/deletion polymorphism in South Indian population with hypertension and chronic kidney disease. J Postgrad Med. 2015;61(4):230-4.

14. Muniandy S, Chan S, Jayapalan J. Null association between ACE gene I/D polymorphism and diabetic nephropathy among multiethnic Malaysian subjects. Indian J Hum Genet. 2010;16(2):78-86.

15. Jiang G, Hu C, Tam CHT, Lau ESH, et al. Genetic and clinical variables identify predictors for chronic kidney disease in type 2 diabetes. Kidney Int. 2016;89(2):411-20.

16. Mrozikiewicz-Rakowska B, Maroszek P, Nehring P, Sobczyk-Kopciol A, et al. Genetic and environmental predictors of chronic kidney disease in patients with type 2 diabetes and diabetic foot ulcer: a pilot study. J Physiol Pharmacol. 2015,66(5):751-61.

17. Chand S, Chue CD, Edwards NC, Hodson J, et al. Endothelial Nitric Oxide Synthase Single Nucleotide Polymorphism and Left Ventricular Function in Early Chronic Kidney Disease. Joles JA, editor. PLoS One. 2015;10(1): e0116160.

18. Kim JH, Shin HD, Park BL, Moon MK, et al. SLC12A3 (solute carrier family 12 member [sodium/chloride] 3) polymorphisms are associated with end-stage renal disease in diabetic nephropathy. Diabetes. 2006,55(3):843-8.

19. Ng DPK, Nurbaya S, Choo S, Koh D, et al. Genetic variation at the SLC12A3 locus is unlikely to explain risk for advanced diabetic nephropathy in Caucasians with type 2 diabetes. Nephrol Dial Transplant. 2008;23(7):2260-4.

20. Ng DPK, Nurbaya S, Choo S, Koh D, et al. Genetic variation at the SLC12A3 locus is unlikely to explain risk for advanced diabetic nephropathy in Caucasians with type 2 diabetes. Nephrol Dial Transplant [Internet]. 2008 May 25 [cited 2015 Nov 4];23(7):22604. Available from: http://www.ncbi.nlm.nih.gov/pubmed/18263 927

\section{Palabras claves:}

GENES; POLIMORFISMOS; ENFERMEDAD RENALCRÓNICA, NEFROPATÍA DIABÉTICA; DIABETES MELLITUS TIPO 2.

\section{Keywords:}

\author{
GENES; POLYMORPHISMS; CHRONIC \\ KIDNEY DISEASE, DIABETIC \\ NEPHROPATHY; DIABETES MELLITUS \\ TYPE 2.
}

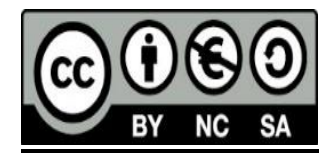




\section{Anexo tablas}

Tabla 1. Secuencia de cebadores, enzimas de restricción y tamaños esperados de los productos de digestión.

\begin{tabular}{|c|c|c|c|c|c|c|}
\hline \multirow{2}{*}{ GEN } & \multirow[t]{2}{*}{ SNP } & \multirow[t]{2}{*}{ Secuencias de cebadores $\left(5^{\prime}>3^{\prime}\right)$} & \multirow{2}{*}{$\begin{array}{l}\text { Tamaño } \\
\operatorname{PCR}(\mathbf{p b})\end{array}$} & \multirow{2}{*}{$\begin{array}{l}\text { Enzima de } \\
\text { Restricción }\end{array}$} & \multicolumn{2}{|c|}{ Fragmentos digeridos $(\mathbf{p b})$} \\
\hline & & & & & Wild-type & Mutado \\
\hline \multirow[b]{2}{*}{$\mathrm{ECA}$} & \multirow[b]{2}{*}{ rs4646994 } & F: CTGGAGACCCCCATCCTTTCT & \multirow{2}{*}{ I: 490} & & & \\
\hline & & $\begin{array}{l}\text { R: } \\
\text { GATGTGGCCATCACATTCGTCAGA }\end{array}$ & & & & \\
\hline \multirow{2}{*}{ CDKAL1 } & \multirow{2}{*}{ rs7756992 } & F: CCTGACTTTTGCCAACCAAA & \multirow{2}{*}{279} & \multirow{2}{*}{ BglII } & \multirow{2}{*}{ A: $201+78$} & \multirow{2}{*}{ G: 279} \\
\hline & & R: TTCATGCAACCAAGAGAGGT & & & & \\
\hline \multirow{2}{*}{ e-NOS } & \multirow{2}{*}{ rs1799983 } & $\begin{array}{l}\text { F: } \\
\text { AAGGCAGGAGACAGTGGATGGA }\end{array}$ & \multirow{2}{*}{248} & \multirow{2}{*}{ MboI } & \multirow{2}{*}{ G: 248} & \multirow{2}{*}{$\mathrm{T}: 163+85$} \\
\hline & & $\begin{array}{l}\text { R: } \\
\text { CCCAGTCAATCCCTTTGGTGCTCA }\end{array}$ & & & & \\
\hline \multirow{2}{*}{ SLC12A3 } & \multirow{2}{*}{ rs11643718 } & F: TTCTACAAGCGCAGGCTTTG & \multirow{2}{*}{626} & \multirow{2}{*}{ AvaI } & $\mathrm{G}: 168+86+$ & A: $168+86$ \\
\hline & & R: GTGCCCTCTGACACATCTAGG & & & $222+149$ & +373 \\
\hline
\end{tabular}


Tabla 2. Parámetros clínicos, bioquímicos y estilos de vida en la población estudiada (media).

\begin{tabular}{|c|c|c|c|}
\hline & \multicolumn{2}{|c|}{ Clasificación de riesgo a desarrollar ERC } & \multirow[b]{2}{*}{ Valor $\mathbf{p}$} \\
\hline & ${ }^{\text {a }}$ Bajo $(n=10)$ & ${ }^{\text {a Alto }(n=15)}$ & \\
\hline $\operatorname{Sexo}(F / M)$ & $4 / 6$ & $10 / 5$ & 0,1882 \\
\hline Edad (Años) & $71,40 \pm 7,95$ & $71,60 \pm 3,57$ & 0,9328 \\
\hline Duración de la diabetes (Años) & $20,60 \pm 15,47$ & $18,60 \pm 15,14$ & 0,7512 \\
\hline Hipertensión (Si/No) & $8 / 2$ & $15 / 0$ & 0,0710 \\
\hline Fumador (Si/No) & $0 / 10$ & $1 / 14$ & 0,4047 \\
\hline $\mathrm{IMC}\left(\mathrm{Kg} / \mathrm{m}^{2}\right)$ & $27,73 \pm 4,99$ & $30,47 \pm 5,77$ & 0,2329 \\
\hline Terapia con Insulina $(\mathrm{Si} / \mathrm{No})$ & $5 / 5$ & $8 / 7$ & 0,8702 \\
\hline Glucemia (mg \%) & $135,80 \pm 67,28$ & $121,09 \pm 62,83$ & 0,5823 \\
\hline HbA1c (\%) & $9,03 \pm 1,80$ & $8,11 \pm 1,78$ & 0,2890 \\
\hline Creatininemia (mg/dl) & $1,02 \pm 0,10$ & $1,47 \pm 0,62$ & $0,0146^{*}$ \\
\hline CKDEPI (ml/min/1,73 m²) & $71,00 \pm 7,06$ & $43,47 \pm 11,38$ & $<0.0001^{*}$ \\
\hline Albuminuria $(\mu \mathrm{g} / \mathrm{min})$ & $24,58 \pm 20,75$ & $33,29 \pm 37,09$ & 0,6479 \\
\hline Colesterol (mg/dl) & $185,14 \pm 49,54$ & $179,55 \pm 30,28$ & 0,7684 \\
\hline Triglicéridos (mg/dl) & $151,00 \pm 105,34$ & $161,55 \pm 76,21$ & 0,8080 \\
\hline HDL colesterol (mg/dl) & $50,67 \pm 12,26$ & $47,50 \pm 11,77$ & 0,6157 \\
\hline LDL colesterol (mg/dl) & $116,50 \pm 44,93$ & $104,60 \pm 35,81$ & 0,5671 \\
\hline ALT (mUI/ml) & $35,50 \pm 26,13$ & $18,22 \pm 5,95$ & 0,1102 \\
\hline AST (mUI/ml) & $31,50 \pm 16,72$ & $17,89 \pm 3,55$ & 0,0586 \\
\hline GGT (mUI/ml) & $47,43 \pm 26,93$ & $27,29 \pm 12,26$ & 0,0969 \\
\hline Presión sistólica (mmHg) & $117.43 \pm 4.43$ & $136.77 \pm 20.39$ & $0.0055^{*}$ \\
\hline Presión diastólica (mmHg) & $68.57 \pm 9.00$ & $76.42 \pm 8.82$ & 0.0806 \\
\hline
\end{tabular}

Los datos están expresados como la media \pm la desviación estándard (DE) o número de pacientes (n). Las diferencias entre los grupos de pacientes ( $\mathrm{Sin} / \mathrm{Con}$ ) Nefropatía fueron comparados usando el t test o el del Chi-cuadrado.

ERC: Enfermedad Renal Crónica

a Bajo: Grado 1 y 2, con Tasa de Filtrado Glomerular estimado igual o mayor a $60 \mathrm{ml} / \mathrm{min} / 1,73 \mathrm{~m}^{2}$.

a Alto: Grado 3a, 3b, 4 y 5: Tasa de Filtrado Glomerular estimado menor a $60 \mathrm{ml} / \mathrm{min} / 1,73 \mathrm{~m}^{2}$.

IMC, índice de masa corporal; HbA1c, hemoglobina glicosilada; HDL, lipoproteína de alta densidad; LDL, lipoproteína de baja densidad; CKDEPI (Chronic Kidney Disease Epidemiology Collaboration); ALT, alanino amino transferasa; AST, aspartato amino transferasa; GGT, gama glutamil transpeptidasa.

Revista Methodo: Investigación Aplicada a las Ciencias Biológicas. Universidad Católica de Córdoba. Jacinto Ríos 571 Bo Gral. Paz. X5004FXS. Córdoba. Argentina. Tel.: (54) 3514517299 / Correo: methodo@ucc.edu.ar / Web: methodo.ucc.edu.ar |ARTICULO ORIGINAL Methodo 2018;3(4):103 -111 
Yang P, Ojeda V, Ruiz-Pecchio A, Soria NW. Rol de variantes genéticas en el desarrollo de Enfermedad Renal Crónica en pacientes con Diabetes Mellitus Tipo 2.

*Estadísticamente significativo.

Tabla 3. Distribución alélica y genotípica junto al equilibrio de Hardy-Weinberg de los diferentes SNPs analizados en la población estudiada.

\begin{tabular}{|l|c|c|c|c|c|c|}
\hline & \multicolumn{3}{|l|}{ Genotipo } & \multicolumn{2}{l|}{ Alelos } & \\
\hline & wt/wt & wt/mut & mut/mut & wt & mut & Equilibrio H-W (p) \\
\hline ECA (rs4646994) & 9 & 8 & 8 & 26 & 24 & 0,0726 \\
\hline CDKAL1 (rs7756992) & 5 & 14 & 6 & 24 & 26 & 0,5425 \\
\hline e-NOS (rs1799983) & 12 & 11 & 2 & 35 & 15 & 0,8118 \\
\hline SLC12A3 (rs11643718) & 4 & 10 & 11 & 18 & 32 & 0,5094 \\
\hline
\end{tabular}

Wt: Wild type

Mut: mutante

H-W: Hardy Weinberg

Tabla 4. Asociación de SNPs presentes en los genes ECA (rs4646994), CDKAL1 (rs7756992), e-NOS (rs1799983) y SLC12A3 (rs11643718) con el riesgo a desarrollar ERC. (Parte I)

\begin{tabular}{|c|c|c|c|c|c|}
\hline Modelo /Genes & Genotipo & $\begin{array}{c}\text { Bajo riesgo } \\
\text { n; }(\%)\end{array}$ & $\begin{array}{c}\text { Alto riesgo } \\
\text { n; }(\%)\end{array}$ & OR (95\% IC) & $\begin{array}{c}\text { Valor } \\
\text { p }\end{array}$ \\
\hline \multicolumn{6}{|l|}{$E C A(r s 4646994)$} \\
\hline \multirow[t]{3}{*}{ Codominante } & $\mathrm{D} / \mathrm{D}$ & $4(40)$ & $5(33,3)$ & 1,00 & \multirow[t]{3}{*}{0,55} \\
\hline & I/D & $4(40)$ & $4(26,7)$ & $0,80(0,12-5,40)$ & \\
\hline & $\mathrm{I} / \mathrm{I}$ & $2(20)$ & $6(40)$ & $2,40(0,30-19,04)$ & \\
\hline \multirow[t]{2}{*}{ Dominante } & $\mathrm{D} / \mathrm{D}$ & $4(40)$ & $5(33,3)$ & 1,00 & \multirow[t]{2}{*}{0,73} \\
\hline & \begin{tabular}{|l|}
$\mathrm{I} / \mathrm{D}-\mathrm{I} / \mathrm{I}$ \\
\end{tabular} & $6(60)$ & $10(66,7)$ & $1,33(0,25-7,01)$ & \\
\hline \multirow[t]{2}{*}{ Recesivo } & D/D-I/D & $8(80)$ & $9(60)$ & 1,00 & \multirow[t]{2}{*}{0,28} \\
\hline & $\mathrm{I} / \mathrm{I}$ & $2(20)$ & $6(40)$ & $2,67(0,41-17,17)$ & \\
\hline \multirow[t]{2}{*}{ Sobredominante } & D/D-I/I & $6(60)$ & $11(73,3)$ & 1,00 & \multirow[t]{2}{*}{0,49} \\
\hline & $\mathrm{I} / \mathrm{D}$ & $4(40)$ & $4(26,7)$ & $0,55(0,10-3,00)$ & \\
\hline Log-aditivo & --- & --- & --- & $1,49(0,55-4,03)$ & 0,43 \\
\hline
\end{tabular}


Tabla 4. Asociación de SNPs presentes en los genes ECA (rs4646994), CDKAL1 (rs7756992), e-NOS (rs1799983) y SLC12A3 (rs11643718) con el riesgo a desarrollar ERC. (Parte II)

\begin{tabular}{|c|c|c|c|c|c|}
\hline Modelo /Genes & Genotipo & Bajo riesgo & Alto riesgo & OR (95\% IC) & $\begin{array}{l}\text { Valor } \\
\mathrm{p}\end{array}$ \\
\hline \multicolumn{6}{|c|}{ CDKAL1 (rs7756992) } \\
\hline \multirow[t]{3}{*}{ Codominante } & $\mathrm{A} / \mathrm{A}$ & $2(20)$ & $3(20)$ & 1,00 & \multirow[t]{3}{*}{0,92} \\
\hline & $\mathrm{A} / \mathrm{G}$ & $6(60)$ & $8(53,3)$ & $0,89(0,11-7,11)$ & \\
\hline & $\mathrm{G} / \mathrm{G}$ & $2(20)$ & $4(26,7)$ & $1,33(0,11-15,70)$ & \\
\hline \multirow[t]{2}{*}{ Dominante } & $\mathrm{A} / \mathrm{A}$ & $2(20)$ & $3(20)$ & 1,00 & \multirow[t]{2}{*}{ NA } \\
\hline & A/G-G/G & $8(80)$ & $12(80)$ & $1,00(0,14-7,39)$ & \\
\hline \multirow[t]{2}{*}{ Recesivo } & $\mathrm{A} / \mathrm{A}-\mathrm{A} / \mathrm{G}$ & $8(80)$ & $11(73,3)$ & 1,00 & \multirow[t]{2}{*}{0,7} \\
\hline & $\mathrm{G} / \mathrm{G}$ & $2(20)$ & $4(26,7)$ & $1,45(0,21-9,98)$ & \\
\hline \multirow[t]{2}{*}{ Sobredominante } & A/A-G/G & $4(40)$ & $7(46,7)$ & 1,00 & \multirow[t]{2}{*}{0,74} \\
\hline & $\mathrm{A} / \mathrm{G}$ & $6(60)$ & $8(53,3)$ & $0,76(0,15-3,86)$ & \\
\hline \begin{tabular}{|l|} 
Log-aditivo \\
\end{tabular} & --- & --- & --- & $1,16(0,35-3,91)$ & 0,81 \\
\hline \multicolumn{6}{|c|}{$e-N O S(r s 1799983)$} \\
\hline \multirow[t]{3}{*}{ Codominante } & $\mathrm{G} / \mathrm{G}$ & $3(30)$ & $9(60)$ & 1,00 & \multirow[t]{3}{*}{0,33} \\
\hline & $\mathrm{G} / \mathrm{T}$ & $6(60)$ & $5(33,3)$ & $0,28(0,05-1,62)$ & \\
\hline & $T / T$ & $1(10)$ & $1(6,7)$ & $0,33(0,02-7,14)$ & \\
\hline \multirow[t]{2}{*}{ Dominante } & $\mathrm{G} / \mathrm{G}$ & $3(30)$ & $9(60)$ & 1,00 & \multirow[t]{2}{*}{0,14} \\
\hline & G/T-T/T & $7(70)$ & $6(40)$ & $0,29(0,05-1,57)$ & \\
\hline \multirow[t]{2}{*}{ Recesivo } & G/G-G/T & $9(90)$ & $14(93,3)$ & 1,00 & \multirow[t]{2}{*}{0,77} \\
\hline & $\mathrm{T} / \mathrm{T}$ & $1(10)$ & $1(6,7)$ & $0,64(0,04-11,63)$ & \\
\hline \multirow[t]{2}{*}{\begin{tabular}{|l|} 
Sobredominante \\
\end{tabular}} & G/G-T/T & $4(40)$ & $10(66,7)$ & 1,00 & \multirow[t]{2}{*}{0,19} \\
\hline & $\mathrm{G} / \mathrm{T}$ & $6(60)$ & $5(33,3)$ & $0,33(0,06-1,75)$ & \\
\hline Log-aditivo & --- & --- & --- & $0,42(0,11-1,62)$ & 0,19 \\
\hline \multicolumn{6}{|c|}{ SLC12A3 (rs11643718) } \\
\hline \multirow[t]{4}{*}{ Codominante } & $\mathrm{G} / \mathrm{G}$ & $2(20)$ & $2(13,3)$ & 1,00 & \multirow[t]{2}{*}{0,66} \\
\hline & $\mathrm{G} / \mathrm{T}$ & $4(40)$ & $6(40)$ & $1,50(0,15-15,46)$ & \\
\hline & $\mathrm{T} / \mathrm{T}$ & $4(40)$ & $7(46,7)$ & $1,75(0,17-17,69)$ & \multirow[t]{2}{*}{0,74} \\
\hline & $\mathrm{T} / \mathrm{T}$ & $4(40)$ & $7(46,7)$ & $1,31(0,26-6,64)$ & \\
\hline \multirow[t]{2}{*}{ Sobredominante } & G/G-T/T & $6(60)$ & $9(60)$ & 1,00 & \multirow[t]{2}{*}{ NA } \\
\hline & $\mathrm{G} / \mathrm{T}$ & $4(40)$ & $6(40)$ & $1,00(0,20-5,12)$ & \\
\hline Log-aditivo & --- & --- & --- & $1,29(0,43-3,91)$ & 0,65 \\
\hline
\end{tabular}

ERC: Enfermedad Renal Crónica.

IC: Intervalo de confianza 95\%. p calculado del test Chi-cuadrado.

$\mathrm{N}$ : número de pacientes

$\%$ : porcentaje de pacientes.

\section{@(1000

